\title{
Shape Optimization of "Pumpkin" Balloons
}

\author{
M. Pagitz * and S. Pellegrino ${ }^{\dagger}$ \\ University of Cambridge, Cambridge, CB2 1PZ, UK
}

\begin{abstract}
This paper is concerned with the optimization of the cutting pattern for the lobes of pumpkin-shaped balloon structures. Pumpkin balloons, currently developed for the NASA Ultra-Long Duration Balloon (ULDB), consist of stiff meridional tendons that constrain a thin plastic film. The present study shows that the maximum stability of pumpkin balloons is reached if their surface area is minimized under a given stress constraint. This results in fully stressed designs that in some cases have a total surface area smaller than a smooth isotensoid surface, without increasing the maximum stresses in the film. The resulting designs are likely to avoid the shape instabilities that have been observed in balloons with a high surface area to volume ratio.
\end{abstract}

\section{Introduction}

Pumpkin (superpressure) balloons, Figure 1(a), use a closed envelope that remains pressurized throughout the day-night cycle. Therefore, the volume of these balloons remains approximately constant and so their float altitude is also constant. Unfortunately, for certain geometries of the lobe cutting pattern these balloons are unstable even at very small pressures, Figure 1(b).

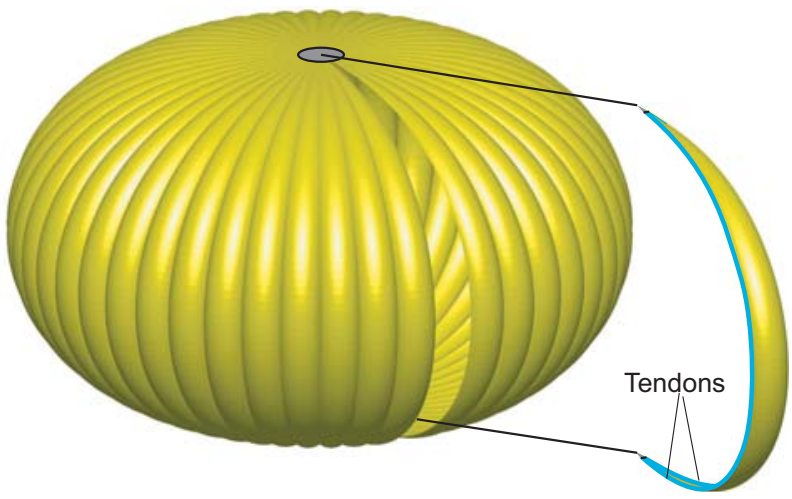

(a)

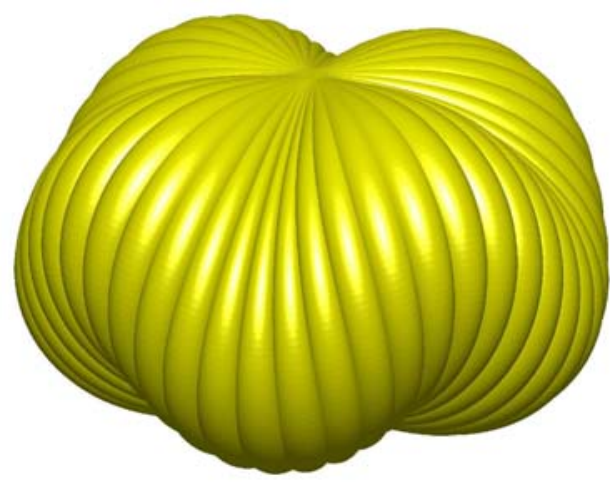

(b)

Figure 1. (a) Concept and (b) typical buckling mode of pumpkin balloon.

Calladine $^{1}$ explained the instability by showing that the volume enclosed by a balloon whose lobes subtend a constant angle increases, if the number of lobes and the bulge formed by the lobes are sufficiently large, for certain small-amplitude inextensional deformations of the balloon. Later, Lennon \& Pellegrino ${ }^{2}$ showed that, again for inextensional deformation modes, the volume enclosed by a balloon whose lobes have uniform transverse radius of curvature, decreases for small deformations but increases for sufficiently large deformations. Both of these analytical models were based on assumed buckling modes and neglected the elastic deformation of the membrane and tendons. The first numerical simulations of the stability of pumpkin balloons were carried out by Wakefield. ${ }^{3}$ Later on, Pagitz \& Pellegrino ${ }^{4}$ considerably simplified

* Research Fellow, Department of Engineering, Trumpington Street.

${ }^{\dagger}$ Professor of Structural Engineering, Department of Engineering, Trumpington Street, Associate Fellow AIAA. 
the computation of the buckling pressure by taking into account the symmetry of the balloons, using closed form solutions for the symmetry transformation matrices. ${ }^{5}$

This paper builds on this recent work and optimizes the cutting patterns of pumpkin balloons such that their stability is maximized for a given stress constraint. It is shown that maximizing the buckling pressure of a balloon is equivalent to minimizing the surface area, and that this results in a fully stressed design. Furthermore it is shown that, compared to existing designs, it is possible to reduce the total surface area without increasing the maximum membrane stresses. Finally, a new cutting pattern design is obtained.

\section{Current Cutting Patterns}

It was shown by Pagitz \& Pellegrino ${ }^{4}$ that the cross-sections of the lobes can be assumed to be circular arcs. Therefore, it is possible to create cutting patterns that have two planes of mirror symmetry and that, when inflated, lead to lobes whose cross-sections have either a constant angle (CA) or a constant radius (CR), Figure 2. Although the difference between $\mathrm{CA}$ and $\mathrm{CR}$ cutting patterns is not very large, balloons made out of these two cutting patterns behave in very different ways. Since these cutting patterns were derived from physical intuition it seems likely that better designs can be obtained from a mathematical optimization approach.
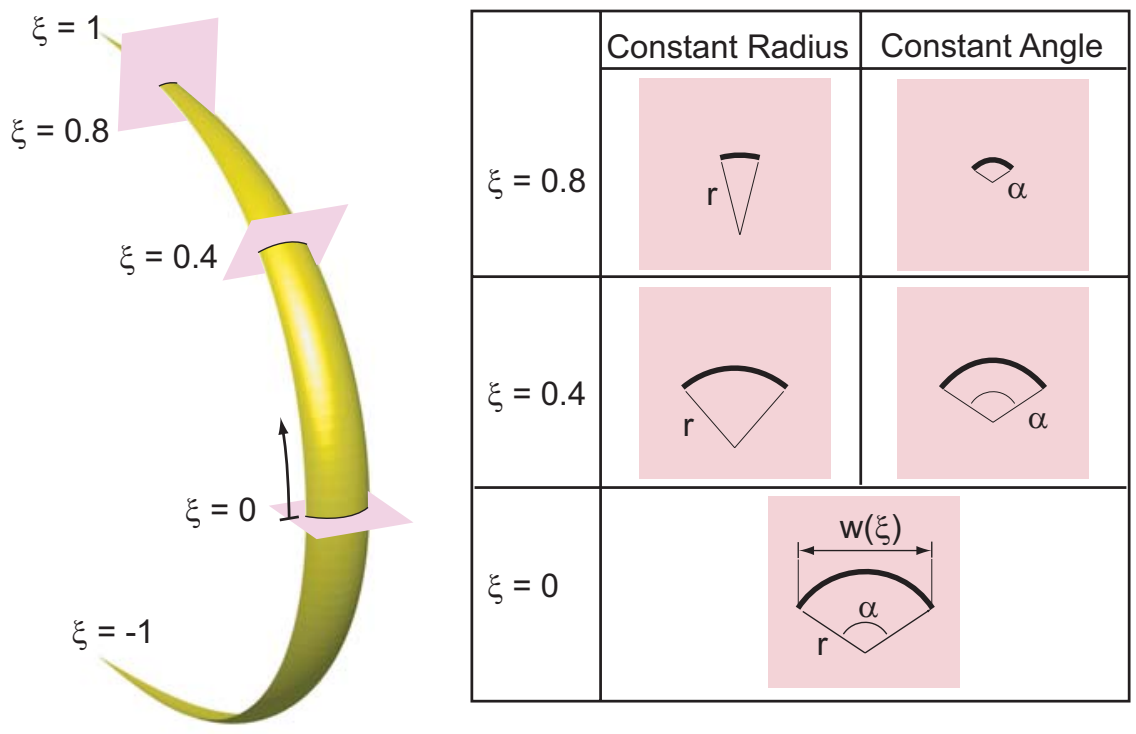

Figure 2. Cross-sections of inflated lobe.

\section{Formulation of Optimization Problem}

Two different objectives are considered in the following in order to maximize the stability of pumpkin balloons. These objectives are

- Minimization of cutting pattern area;

- Modification of cutting pattern shape such that a certain eigenvalue is maximized at a given differential pressure $p$.

Both objectives were investigated, each coupled with a maximum stress constraint that limits the resulting membrane stresses. The maximum stress constraint is defined in terms of the von Mises stress

$$
\sigma_{v M}=\sqrt{\sigma_{1}^{2}+\sigma_{2}^{2}-\sigma_{1} \sigma_{2}}
$$

where $\sigma_{1,2}$ are the principal stresses. It was found that both objectives result in similar cutting patterns. Therefore, since minimizing the cutting pattern area is faster than maximizing the eigenvalue of a balloon, 
all results given in the following are based on the former.

\section{Shape Functions for Cutting Patterns}

Bernstein polynomials are used as shape functions to represent the cutting patterns. The $n+1$ Bernstein polynomials ${ }^{6}$ of degree $n$ are defined as

$$
B_{k, n}(t)=\left(\begin{array}{c}
n \\
k
\end{array}\right) t^{k}(1-t)^{n-k}
$$

with $t \in[0,1]$; the binomial coefficient $\left(\begin{array}{c}n \\ k\end{array}\right)$ is

$$
\left(\begin{array}{l}
n \\
k
\end{array}\right)=\frac{n !}{(n-k) ! k !}
$$

Hence, the polynomials for $n=0,1,2$ are

$$
\begin{aligned}
& n=0: \quad B_{0,0}(t)=1 ; \quad n=1: \quad B_{0,1}(t)=1-t, \quad n=2: \quad B_{0,2}(t)=(1-t)^{2} \\
& B_{1,1}(t)=t ; \quad B_{1,2}(t)=2(1-t) t \\
& B_{2,2}(t)=t^{2} \text {. }
\end{aligned}
$$

Figure 3 shows plots of the polynomials of degree up to $n=5$. Bernstein polynomials have a number of useful properties. In particular, they satisfy symmetry

$$
B_{k, n}(t)=B_{n-k, n}(1-t)
$$

and the sum of all polynomials of degree $n$ is equal to 1 , i.e.

$$
\sum_{k=0}^{n} B_{k, n}(t)=1
$$

Especially important is the next property, that is used later on to simplify the shape optimizations. The areas of the polynomials of degree $n$ in the interval $t \in[0,1]$ are

$$
\int_{t=0}^{1} B_{k, n}(t)=\int_{t=0}^{1}\left(\begin{array}{c}
n \\
k
\end{array}\right) t^{k}(1-t)^{n-k}=\frac{1}{n+1}
$$

and therefore identical. This property is useful since it leads to a linear objective if the area of cutting patterns is to be minimized.

In order to get useful results from the optimization it is essential that one chooses shape functions that have a sufficient approximation power to describe the cutting patterns in the vicinity of the optimum. Since there exists no closed form solution for optimal cutting patterns it is advisable to study the approximation power of Bernstein polynomials for existing cutting patterns. This is done by interpolating a CR cutting pattern of a balloon with 145 lobes and a subtended angle of $118^{\circ}$ at the equator with the method of least squares. In the following the equations are derived for a least squares interpolation of a set of given points, with the help of Bernstein polynomials.

We define the matrix $\mathbf{C}$, of size $p \times m$, containing the values of the single polynomials at selected interpolation points $t_{1} \ldots t_{p}$

$$
\mathbf{C}=\left(\begin{array}{ccc}
B_{1, n}\left(t_{1}\right) & \cdots & B_{m, n}\left(t_{1}\right) \\
\vdots & \vdots & \vdots \\
B_{1, n}\left(t_{p}\right) & \cdots & B_{m, n}\left(t_{p}\right)
\end{array}\right)
$$

we also define the vector $\mathbf{d}=-\left(\begin{array}{lll}y\left(t_{1}\right) & \cdots & y\left(t_{p}\right)\end{array}\right)^{T}$ consisting of the ordinates at the interpolation points. 


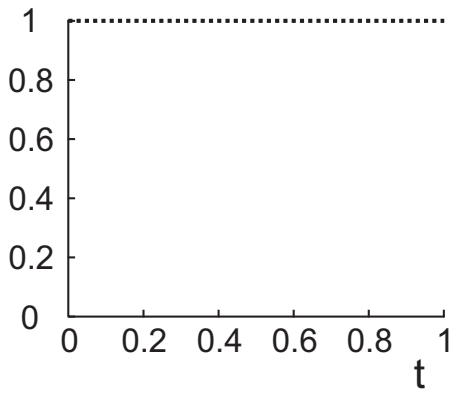

(a) $n=0$

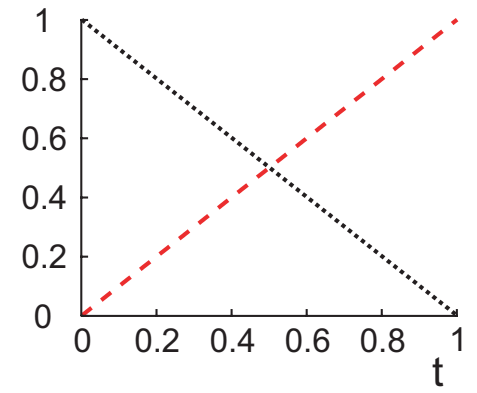

(b) $n=1$

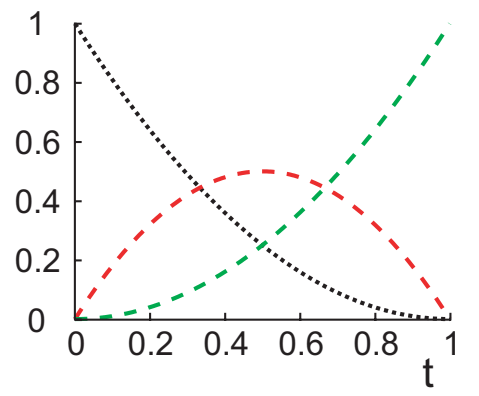

(c) $n=2$

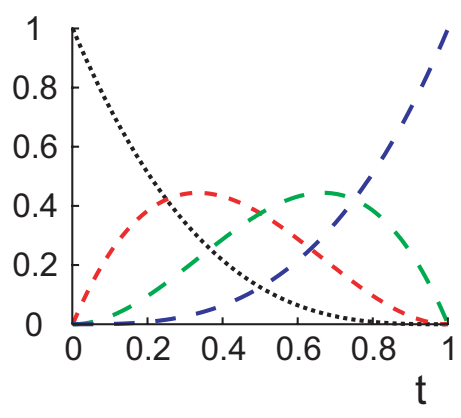

(d) $n=3$

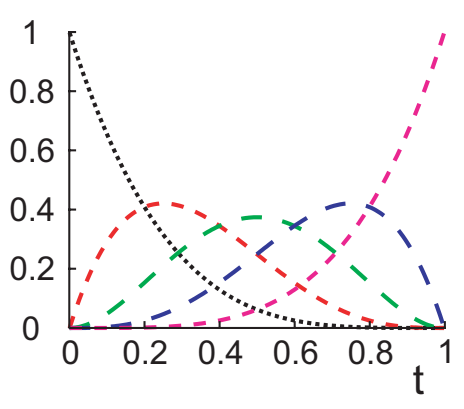

(e) $n=4$

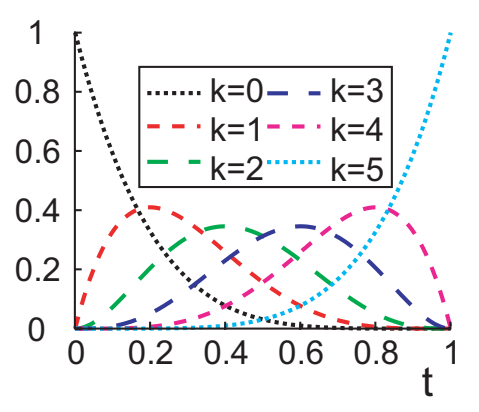

(f) $n=5$

Figure 3. Bernstein polynomials of degree $n \in[0,5]$.

The residual of the interpolation at the points $t_{1}, \ldots, t_{p}$ is

$$
\mathbf{r}=\mathbf{C v}+\mathbf{d}
$$

where the vector $\mathbf{v}$ contains the optimization variables (control points). It can be computed by minimizing the equation

$$
\mathbf{r}^{T} \mathbf{r}=(\mathbf{C v}+\mathbf{d})^{T}(\mathbf{C v}+\mathbf{d})=\mathbf{v}^{T} \mathbf{C}^{T} \mathbf{C v}+2\left(\mathbf{C}^{T} \mathbf{d}\right)^{T} \mathbf{v}+\mathbf{d}^{T} \mathbf{d} .
$$

With the abbreviations $\mathbf{A}=\mathbf{C}^{T} \mathbf{C}$ and $\mathbf{b}=\mathbf{C}^{T} \mathbf{d}$ this finally results in

$$
\mathbf{r}^{T} \mathbf{r}=\mathbf{v}^{T} \mathbf{A} \mathbf{v}+2 \mathbf{b}^{T} \mathbf{v}+\mathbf{d}^{T} \mathbf{d}=F(t)
$$

For the error $F(t)$ to be minimal it is necessary that $\nabla F(t)=0$, which leads to the linear system of equations

$$
\mathbf{A v}+\mathbf{b}=0 .
$$

Figure 4(a) shows interpolations of one side of the cutting pattern, for $n=5$. The red dotted line illustrates the interpolation of the complete curve and the green dotted line the interpolation of only one half. Figure 4(b) shows the error of the interpolation. It becomes obvious that by making use of reflection symmetry the interpolation has a much higher accuracy for the same number of optimization variables. It can be seen that an interpolation of degree $n \geq 5$ gives very good results. Hence, only one half of the upper part of the cutting pattern is interpolated in the following and the complete curve is obtained by reflection. The half-width has the following expression

$$
w(t)=\sum_{k=0}^{n} P_{k} B_{k, n}(t) \quad \text { for } t \in[0,1]
$$

where $P_{k}$ are constant factors that multiply each polynomial, and are contained in the vector $\mathbf{v}$ in Equation 9.

Since the width at the apex and the tangent of the cutting pattern at the mid point are zero it is possible to reduce the number of unknowns,

$$
w(t=0)=0 \text {, hence } P_{0}=0
$$

\section{4 of 17}


and

$$
w^{\prime}(t=1)=0: \quad \frac{\partial w(1)}{\partial t}=\sum_{k=0}^{n} P_{k} \frac{\partial B_{k, n}(1)}{\partial t}=0 \quad \rightarrow \quad P_{n-1}=P_{n}
$$

Hence the number of optimization variables reduces to $n-1$.

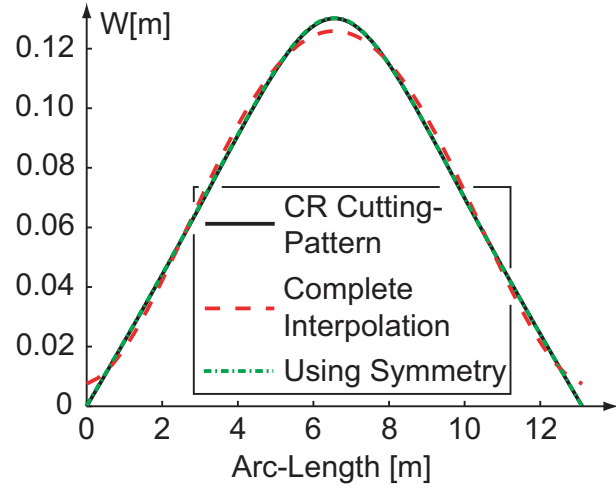

(a) Interpolation.

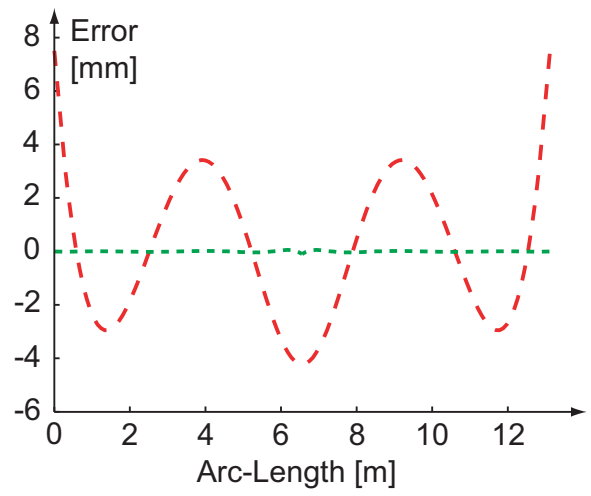

(b) Error of interpolation.

Figure 4. Interpolation of CR cutting pattern with Bernstein polynomials of degree 5.

\section{Optimization Algorithm}

Since the objectives are either linear or mildly non-linear, optimization algorithms based on second-order information are not applicable. A further requirement on the algorithm is that a non-linear maximum stress constraint has to be taken into account. The shape optimization and line search algorithms that have been implemented are presented in the following.

\section{A. Method of Feasible Directions}

A gradient based algorithm that is capable of dealing with highly non-linear constraints is chosen for the shape optimization. An algorithm that fits into this scheme is the "Method of Feasible Directions" by Zoutendijk. ${ }^{7}$ A version of this algorithm is presented in the following for $n$ optimization variables and one constraint.

The objective $f(\mathbf{v})$ and the constraint $g(\mathbf{v})$ are functions of the $n$ optimization variables $\mathbf{v}=\left[\begin{array}{lll}v_{1} & \ldots & v_{n}\end{array}\right]^{T}$. Hence, the gradients $\partial f / \partial \mathbf{v}$ and $\partial g / \partial \mathbf{v}$ can be written as

$$
\frac{\partial f}{\partial \mathbf{v}}=\left[\begin{array}{llll}
\frac{\partial f}{\partial v_{1}} & \frac{\partial f}{\partial v_{2}} & \ldots & \frac{\partial f}{\partial v_{n}}
\end{array}\right]^{T} \text { and } \frac{\partial g}{\partial \mathbf{v}}=\left[\begin{array}{llll}
\frac{\partial g}{\partial v_{1}} & \frac{\partial g}{\partial v_{2}} & \ldots & \frac{\partial g}{\partial v_{n}}
\end{array}\right]^{T} .
$$

The equation of an hyperplane passing through point $\mathbf{v}$, where the change of objective is zero, can be written as

$$
\Delta f=\frac{\partial f}{\partial \mathbf{v}}^{T} \Delta \mathbf{v}=\frac{\partial f}{\partial v_{1}} \Delta v_{1}+\frac{\partial f}{\partial v_{2}} \Delta v_{2}+\ldots+\frac{\partial f}{\partial v_{n}} \Delta v_{n}=0
$$

and solving for $\Delta v_{n}$ gives

$$
\Delta v_{n}=-\frac{\frac{\partial f}{\partial v_{1}} \Delta v_{1}+\frac{\partial f}{\partial v_{2}} \Delta v_{2}+\ldots+\frac{\partial \mathbf{f}}{\partial v_{n-1}} \Delta v_{n-1}}{\frac{\partial f}{\partial v_{n}}} .
$$

For movements in this hyperplane the gradient of the constraint results in

$$
\Delta g=\frac{\partial g}{\partial v_{1}} \Delta v_{1}+\ldots+\frac{\partial g}{\partial v_{n-1}} \Delta v_{n-1}-\frac{\frac{\partial g}{\partial v_{n}}\left(\frac{\partial f}{\partial v_{1}} \Delta v_{1}+\ldots+\frac{\partial f}{\partial v_{n-1}} \Delta v_{n-1}\right)}{\frac{\partial f}{\partial v_{n}}}
$$


where Equation 18 is introduced to keep the objective fixed. The direction where the constraint has a maximal gradient thus becomes

$$
\frac{\partial \Delta g}{\partial \mathbf{v}}=\left[\begin{array}{llll}
\frac{\partial g}{\partial v_{1}}-\frac{\frac{\partial g}{\partial v_{n}} \frac{\partial f}{\partial v_{1}}}{\frac{\partial f}{\partial v_{n}}} & \ldots & \frac{\partial g}{\partial v_{n-1}}-\frac{\frac{\partial g}{\partial v_{n}} \frac{\partial f}{\partial v_{n-1}}}{\frac{\partial f}{\partial v_{n}}} & 0
\end{array}\right]^{T}
$$

and $v_{n}$ can be obtained by inserting Equation 20 into Equation 18

$$
v_{n}=\frac{\frac{\partial g}{\partial v_{n}}{\frac{\partial f}{\partial v_{1}}}^{2}+\ldots+\frac{\partial g}{\partial v_{n}}{\frac{\partial f}{\partial v_{n-1}}}^{2}-{\frac{\partial f}{\partial v_{1}}}_{\frac{\partial f}{\partial v_{n}}} \frac{\partial g}{\partial v_{1}}-\ldots-\frac{\partial f}{\partial v_{n-1}} \frac{\partial f}{\partial v_{n}} \frac{\partial g}{\partial v_{n-1}}}{{\frac{\partial f}{\partial v_{n}}}^{2}} .
$$

Similar expressions for a hyperplane on which the change of constraint is zero can be obtained by exchanging $f$ with $g$. The directions of steepest descent for both hyperplanes are illustrated in Figure 5 and it can be seen that they border a region that is usable - the objective is decreased or stays the same - and feasible - the constraint is decreased or stays the same - for vectors of infinitesimally small length that are located in this region.

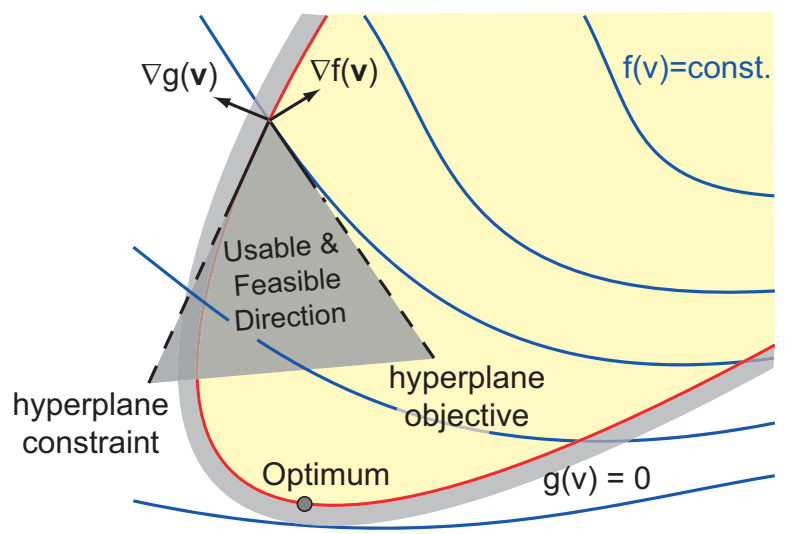

Figure 5. Illustration of Method of Feasible Directions.

Many different methods have been proposed to determine the angle of the direction with respect to the steepest descent in one of the hyperplanes. A detailed discussion and references to the major papers in this field can be found in Reference. ${ }^{8}$ Throughout this paper, to simplify matters, the direction of the single iteration steps is located along the steepest descent of the hyperplanes. Starting from a feasible solution, the actual result is improved by moving in a direction in which the constraint is unchanged while the objective is improved. If the constraint is violated then the following iteration uses the other hyperplane that leaves the objective unchanged (for infinitesimally small displacements). Close to the optimum - defined by the Karush $^{9}$-Kuhn-Tucker ${ }^{10}$ condition - the directions of steepest descent of both hyperlanes become identical, hence the magnitude of their scalar product can be used as a convergence criterion.

\section{B. Line Search}

The previous section has shown how to compute the direction of a new iteration step, however the step length is still unknown. It can be determined with the help of the so-called "line search algorithms", which are able to find an extremum of a function of one variable and also can find the value of the variable for which the function has a prescribed value. Two of the most popular line search algorithms have been considered; their advantages and drawbacks are discussed next.

Since the value of the function and its derivative are known from the optimization at the origin of the search vector, polynomial approximations are often an excellent choice to find an extremum with a minimum number of function evaluations. However, these methods have the disadvantage that the accuracy of the interpolations cannot be guaranteed and for highly non-linear problems it might be quite poor. Hence, the rate of convergence for methods that are based on curve-fitting is not known. ${ }^{8}$ The golden section method ${ }^{11}$ 
is another technique that is able deal with highly non-linear functions; furthermore, the rate of convergence is known.

Let $f(x)$ be a convex function with a minimum in the interval $x \in[0,1]$; the function values $f(0)$ and $f(1)$ are known. If this interval is divided at a point $a$, such that $a \geq b=1-a$, where the function has the value $f(a)$ then the initial state of the line search is obtained, as shown in Figure 6 . The function has a minimum in the given interval if and only if $f(a)<f(0) \wedge f(a)<f(1)$.

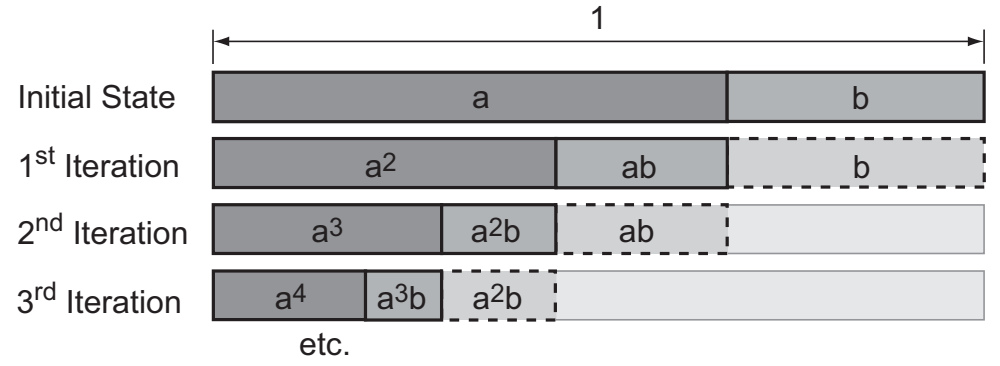

Figure 6. Scheme of golden section method.

To determine a new, smaller interval that contains the minimum of the function it is necessary to compute a fourth value. The location of this value is chosen in the larger interval $[0, a]$ - to guarantee a better distribution - and is determined such that the ratio between the larger and smaller subintervals is again equal to $a / b$ which results in $f\left(a^{2}\right)$ (see $1^{\text {st }}$ iteration of Figure 6). If $f\left(a^{2}\right)<f(a)$ then the minimum can be found in the interval $[0, a]$, if $f\left(a^{2}\right)>f(a)$ it is in the interval $\left[a^{2}, 1\right]$ and for $f\left(a^{2}\right)=f(a)$ both intervals contain the minimum. Assuming $f\left(a^{2}\right)+f(a b) \geq f(a b)+f(b)$ then it is more likely that the minimum is located in the larger interval $\left[0, a^{2}+a b\right]$. Figure 6 shows, under the assumption that the minimum is always located in the larger subinterval, the next two iteration steps to determine the position of the minimum. Hence, the length $l$ of the subinterval after the $n^{\text {th }}$ iteration becomes

$$
l \leq a^{n+1}+a^{n} b .
$$

For the case where $f\left(a^{n+1}\right)+f\left(a^{n} b\right)=f\left(a^{n} b\right)+f\left(a^{n-1} b\right)$ the following convergence rate can be guaranteed

$$
l=a^{n+1}+a^{n} b .
$$

Therefore,

$$
a=\frac{-1+\sqrt{5}}{2} ; \quad b=\frac{3-\sqrt{5}}{2}
$$

and hence

$$
\tau=\frac{a}{b}=\frac{-1+\sqrt{5}}{3-\sqrt{5}}=\frac{1+\sqrt{5}}{2}=1.618033989 \ldots
$$

is the golden ratio.

As an example, we discuss the performance of the golden section method and polynomial interpolation in finding the minimum of the highly non-linear function

$$
f(x)=-1-20 x+e^{6 x}
$$

Figure 7 shows this function, together with polynomial interpolations of degree $n=2,3,4$ that make use of the gradient $f^{\prime}(0)$ and the function values at $f(0)$ and $f(1)$. The remaining information needed for the interpolations is computed according to a grid that is identical to the one obtained by the golden section algorithm. It can be seen that the minima predicted for the three values of $n$ are quite poor and clearly inadequate for an optimization algorithm. Increasing the polynomial degree is not advisable due to possible oscillations. In contrast, the first five iterations of the golden section method are shown at the top of Figure 7. It becomes obvious that much better and more reliable results are obtained with this method. However, this comes at the cost of a larger number of function evaluations. 


\section{A. Small Scale Balloons}

Small scale balloons with a diameter of $10 \mathrm{~m}$ and different number of lobes $n=16,80,145$ have been optimized. The optimization was done at a differential pressure of $p=500 \mathrm{~Pa}$ and the maximum allowed von Mises stresses in the membrane were set equal to the maximum stresses that occur in a CR cutting pattern with a subtended angle of $118^{\circ}$. Note that the subtended angles of the optimized cutting patterns are not fixed but are in fact a result of the optimization itself.

Figure 8(a) shows the stress distribution in the cutting patterns before and after the optimization. It should be noted that in the figure the width of each CR cutting pattern has been scaled to the same width, and the width of the corresponding optimized pattern has been scaled proportionally. It can be seen that the stresses in the optimized cutting patterns are nearly uniform. Furthermore it should be noted that the optimized cutting patterns have a much smaller area than the corresponding CR designs. This effect is especially pronounced for designs with a small number of lobes. For a large number of lobes the reduction ratio of the width converges to a constant value. This is illustrated in Figure 8(b) where the scaled cutting patterns are plotted together with a CR design.

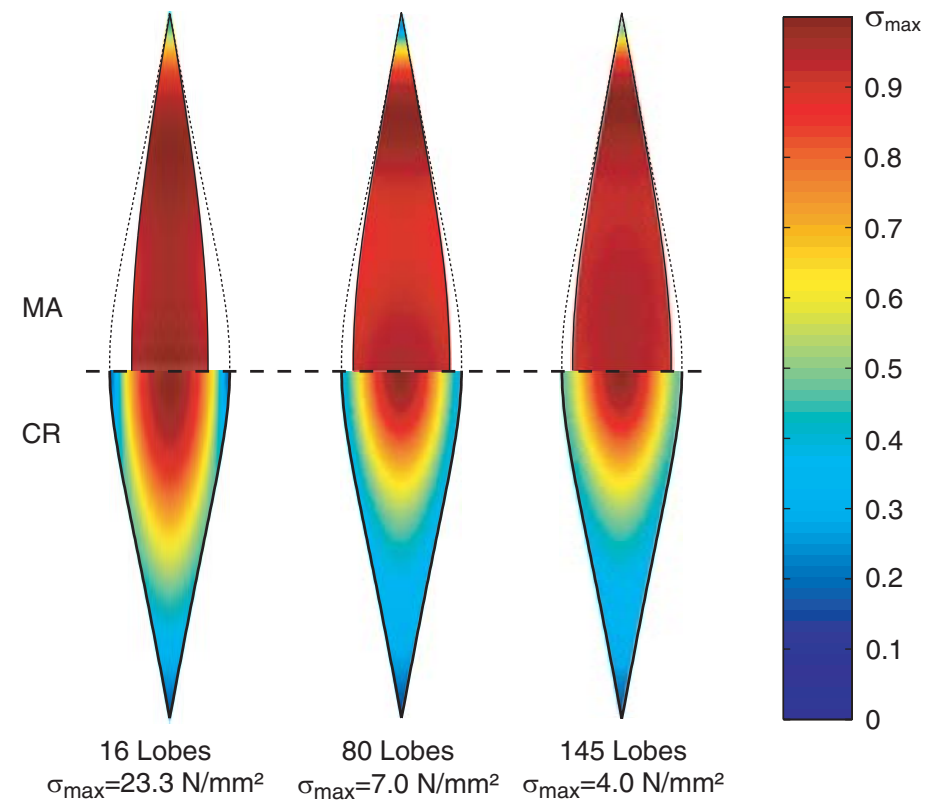

(a) Von Mises stresses.

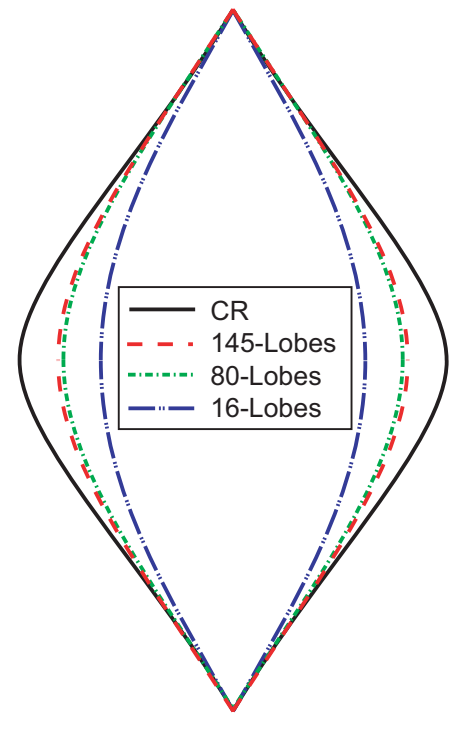

(b) Shape of cutting patterns.

Figure 8. Comparison between minimum area (MA) and CR cutting patterns of small scale balloons at $p=500 \mathrm{~Pa}$.

Figure 9 shows the shape of the tendons of balloons with $\mathrm{CR}$ and optimized cutting patterns. It is interesting to note that the shapes of the optimized tendons, particularly for the smaller values of $n$, are closer to a sphere. Since the stresses in the membrane of a 16-lobe balloon with a CR cutting pattern are relatively high the optimization generates a nearly spherical balloon with an optimum ratio of surface area to enclosed volume. Figure 10 shows a 80-lobe balloon before and after the optimization together with the von Mises stresses in one lobe. It can be seen that the surface of the optimized balloon is much less lobed due to the smaller cutting pattern area. Nevertheless the maximum stresses in both designs are identical. The reason why it is possible to create cutting patterns with smaller areas and identical maximum membrane stresses than conventional CR designs is because of the large strains that occur at the equator due to the incompatibility between a flat and doubly curved surface. See Pagitz \& Pellegrino ${ }^{4}$ for a detailed discussion. One of the many advantages of a fully stressed design is illustrated in Figure 11. It can be seen that the optimized designs are clearly more stable than their CA and CR counterparts. This is especially remarkable since they have identical maximum von Mises stresses. 


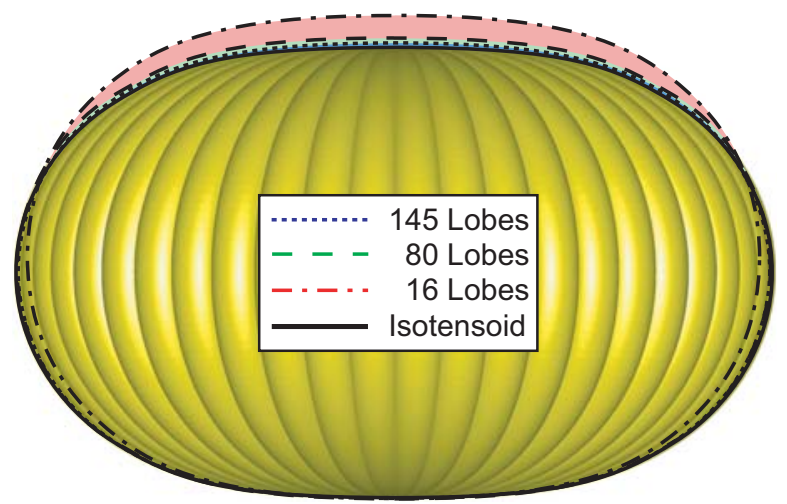

(a) CR cutting patterns.

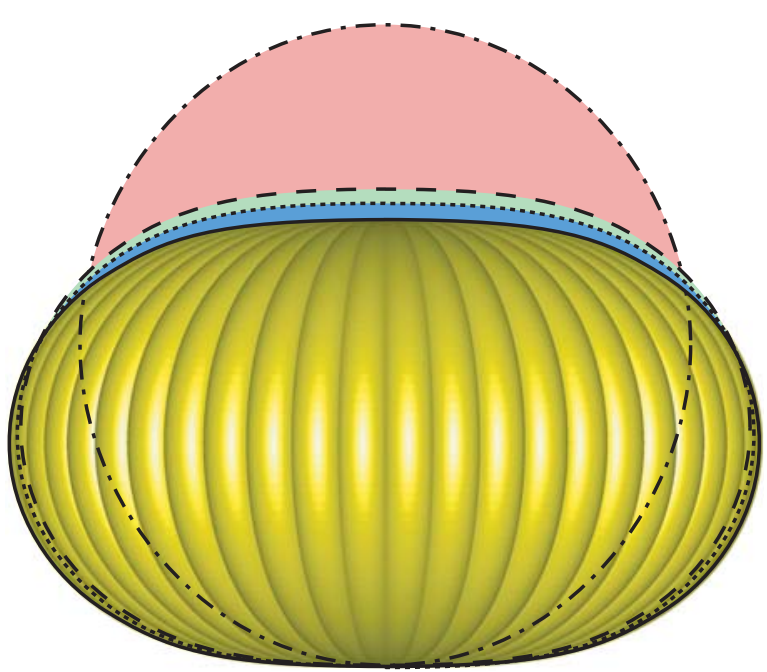

(b) Optimized cutting patterns.

Figure 9. Shape of tendons for CR and optimized cutting patterns of small scale balloons at $p=500 \mathrm{~Pa}$.

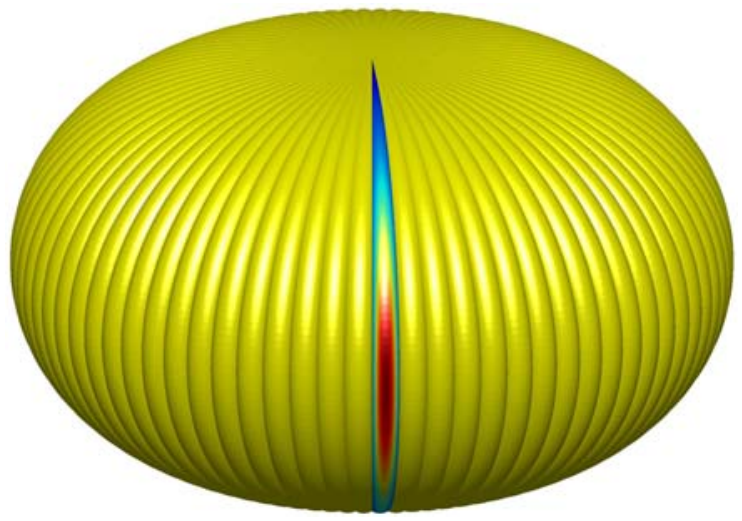

(a) CR 80 lobes

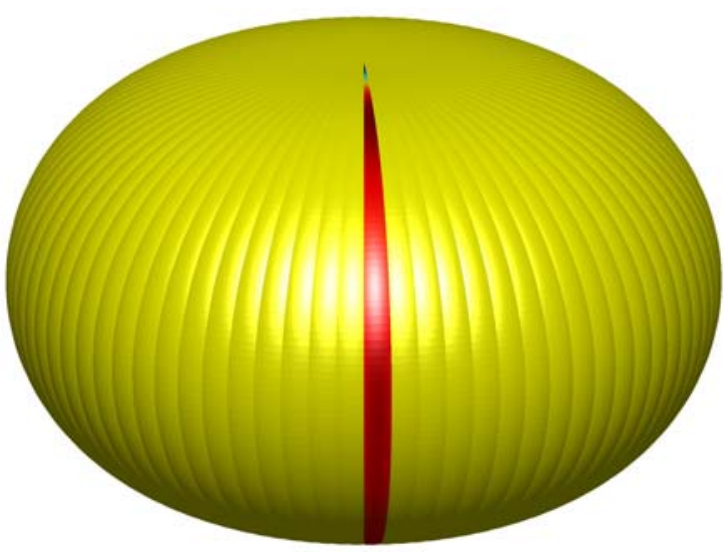

(b) Optimized 80 lobes

Figure 10. Von Mises stresses of small scale balloon based on CR and optimized cutting patterns at $p=500 \mathrm{~Pa}$. 


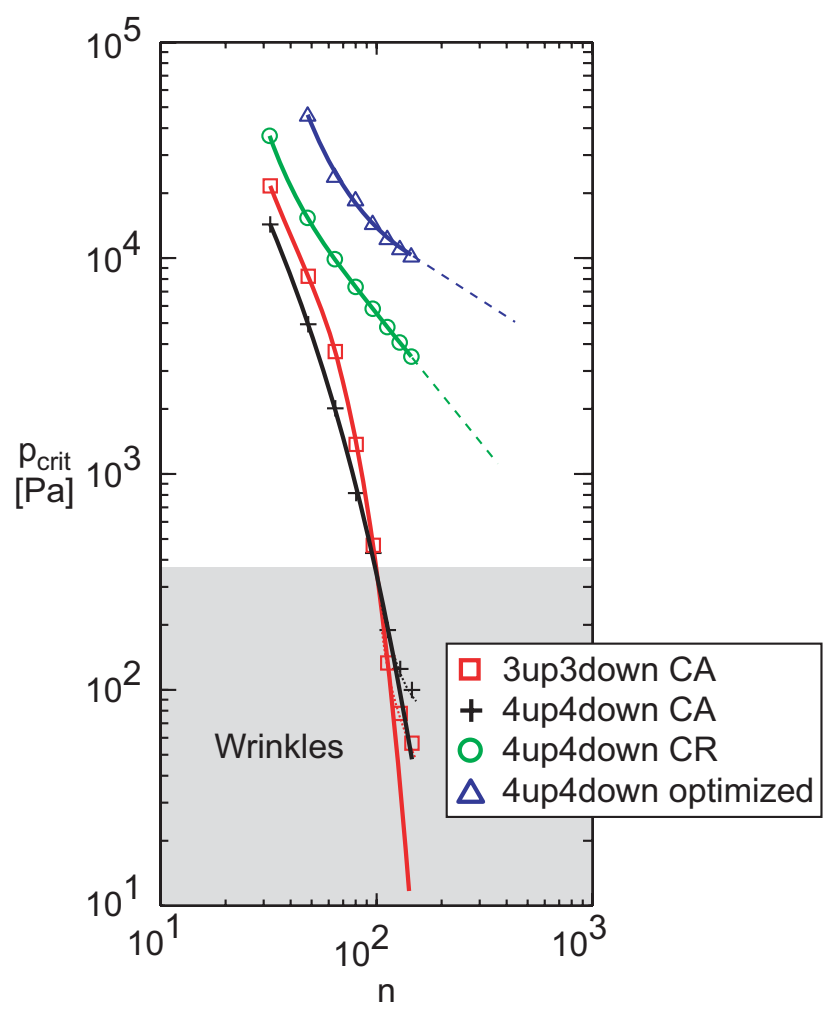

Figure 11. Buckling pressure vs. number of lobes for small scale balloons with CA, CR and optimized cutting patterns, and associated mode shapes. 


\section{B. Flight-Size Balloons}

It has been shown for small scale balloons that optimizing the cutting pattern can considerably increase the buckling pressure. This section extends the investigation to flight-size balloons. Figure 12 shows the von Mises stresses of an original and optimized cutting pattern, for a flight-size balloon at $p=200 \mathrm{~Pa}$. It can be seen that, as already in the case of the small scale balloons, the von Mises stresses are nearly uniform after the optimization. Furthermore the width of the cutting pattern reduces considerably.

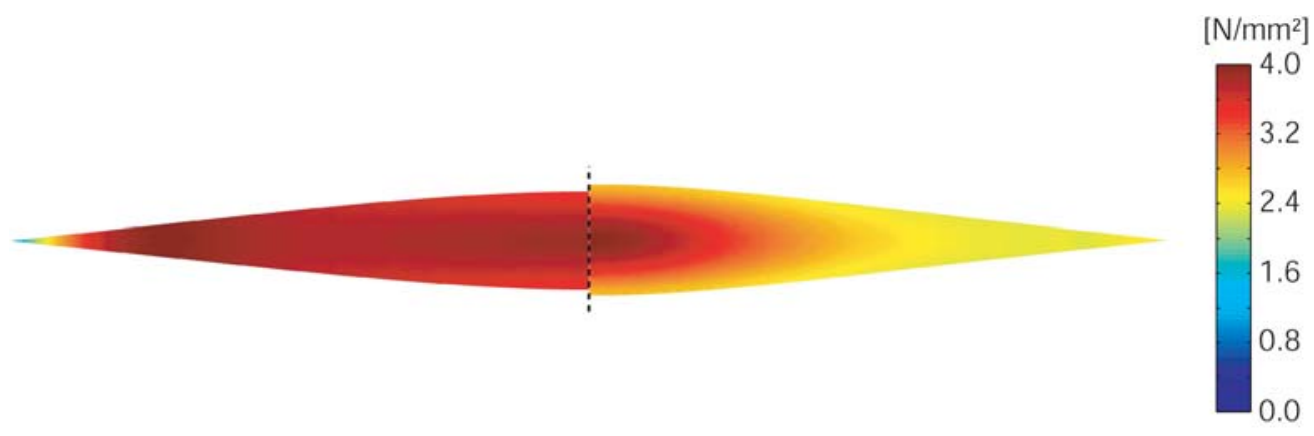

Figure 12. Von Mises stresses of original and optimized cutting pattern of flight size balloon at $p=200 \mathrm{~Pa}$.

A comparison of the corresponding maximum stresses and cutting pattern areas $(A$ is the unstressed surface area of a given design and $A_{S}$ is the area of a sphere) before and after the optimization is presented in Table 2. Note that the surface area decreases considerably, as in the previous examples. Another interesting result is that the optimized cutting pattern for a balloon where the tendons are prestrained during construction results in a balloon with $p_{\text {crit }}=2,117 \mathrm{~Pa}$ and a further slight reduction of the total surface area. Hence the effect that a prestress in the tendons reduces the buckling pressures, observed for CR designs by Pagitz \& Pellegrino, ${ }^{4}$ is not seen in optimized cutting patterns.

\begin{tabular}{lcccccc} 
& & Sphere & Isotensoid & CR & MA & MA with $\varepsilon_{0}=0.3 \%$ \\
\hline Volume & {$\left[\mathrm{m}^{3}\right]$} & \multicolumn{5}{c}{$175,731(\sim 6 \mathrm{mcf})$} \\
Radius & {$[\mathrm{m}]$} & 34.75 & 40 & 40 & 40 & 40 \\
$\left(A-A_{S}\right) / A_{S}$ & {$[\%]$} & 0 & 4.1 & 13.4 & 5.9 & 4.5 \\
Maximum $\sigma_{1}$ & {$\left[\mathrm{~N} / \mathrm{mm}^{2}\right]$} & 72.4 & $\infty$ & 4.7 & 4.4 & 4.6 \\
Maximum $\sigma_{2}$ & {$\left[\mathrm{~N} / \mathrm{mm}^{2}\right]$} & 72.4 & 0 & 2.5 & 3.8 & 3.6 \\
Maximum von Mises stress & {$\left[\mathrm{N} / \mathrm{mm}^{2}\right]$} & 72.4 & $\infty$ & 4.0 & 4.0 & 4.0 \\
$p_{\text {crit }}$ & {$[\mathrm{Pa}]$} & $\infty$ & 0 & 734 & 1,953 & 2,117
\end{tabular}

Table 2. Comparison of cutting patterns for flight-size balloons, before and after optimization.

Figure 13 is a plot the membrane stresses along the centreline of the gore, for a CR flight-size balloon and its optimized counterpart, at a differential pressure of $200 \mathrm{~Pa}$. It should be noted that, in both cases, the maximum von Mises stress is $4 \mathrm{~N} / \mathrm{mm}^{2}$. It can be seen that, for the optimized cutting pattern, both principal stresses are more uniform along the arc-length. Note that it is not possible to reduce the maximum von Mises stress in the membrane for a given number of lobes and a differential pressure arbitrarily by increasing the width of the cutting patterns. The reason for this is that the longitudinal stress peak at the equator cannot be changed significantly by modifying the width of the cutting pattern.

Figure 14 compares $\mathrm{CR}$ and optimized cutting patterns at $p=200 \mathrm{~Pa}$. Figure $14(\mathrm{a})$ is a plot of the ratio between arc length (measured on the inflated lobe) and cord length of the lobe cross-section, along the lobe. This ratio is much smaller for the optimized cutting pattern due to the reduced width. It is interesting to consider also the radius of the inflated lobes, plotted in Figure 14(b). As expected the CR cutting pattern produces a nearly uniform radius; however, the optimized result is quite different. The reason for the reduction towards the equator is the high longitudinal stresses due to the geometric incompatibility, hence the stresses in the transverse direction have to be reduced in this region by decreasing the radius. 


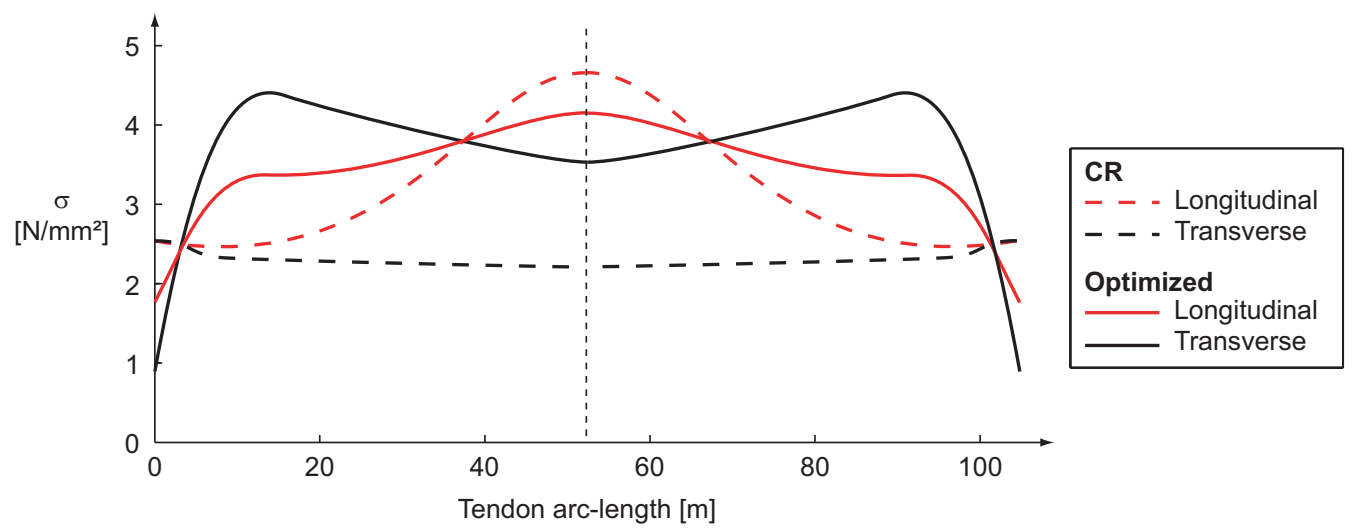

Figure 13. Principal stresses along lobe centreline for CR and optimized flight-size balloon, at $p=200 \mathrm{~Pa}$.

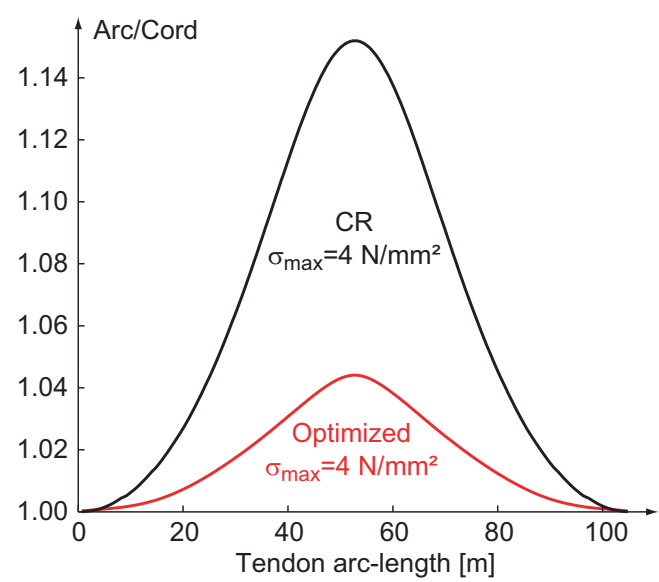

(a) Ratio between arc length and cord length.

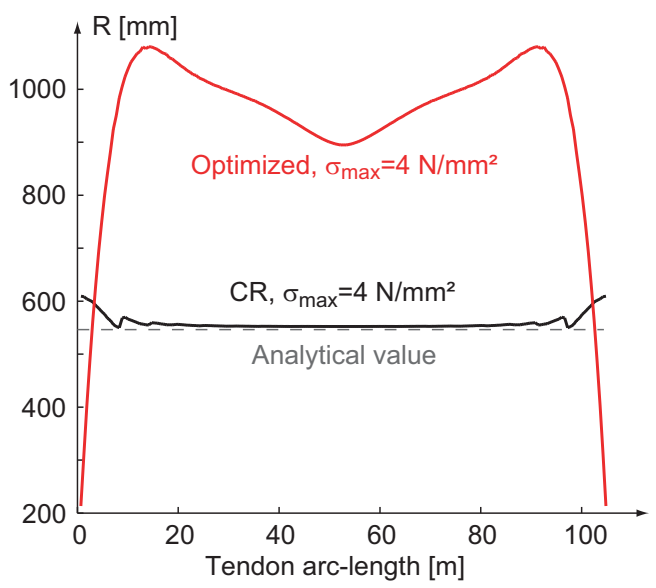

(b) Radius.

Figure 14. Comparison of $\mathbf{C R}$ and optimized cutting pattern of flight-size balloon at $p=200 \mathrm{~Pa}$. 
Figure 15 shows a plot of deformed and undeformed surface areas of balloons with CR and optimized cutting patterns, together with the maximum von Mises stress in each case. The deformed surface areas and the stresses have been computed for a differential pressure of $200 \mathrm{~Pa}$. A striking result is that the optimized cutting patterns have a considerably smaller surface area than the conventional constant radius designs, at comparable or even smaller maximum von Mises stresses. This is even more surprising since the undeformed surface areas of some optimized balloons are smaller than the area of the corresponding isotensoid of identical volume. This observation may be advantageous in the design of pumpkin balloons that avoid undesired equilibrium configurations during ascent. Furthermore it should be noted that the pressure-induced increase in surface area of the optimized designs is approximately proportional to the maximum allowed membrane stress, because the stress distribution is nearly uniform. Another nice result is that the pressurized surface area with a given maximum von Mises stress at the smallest possible number of lobes is roughly constant.

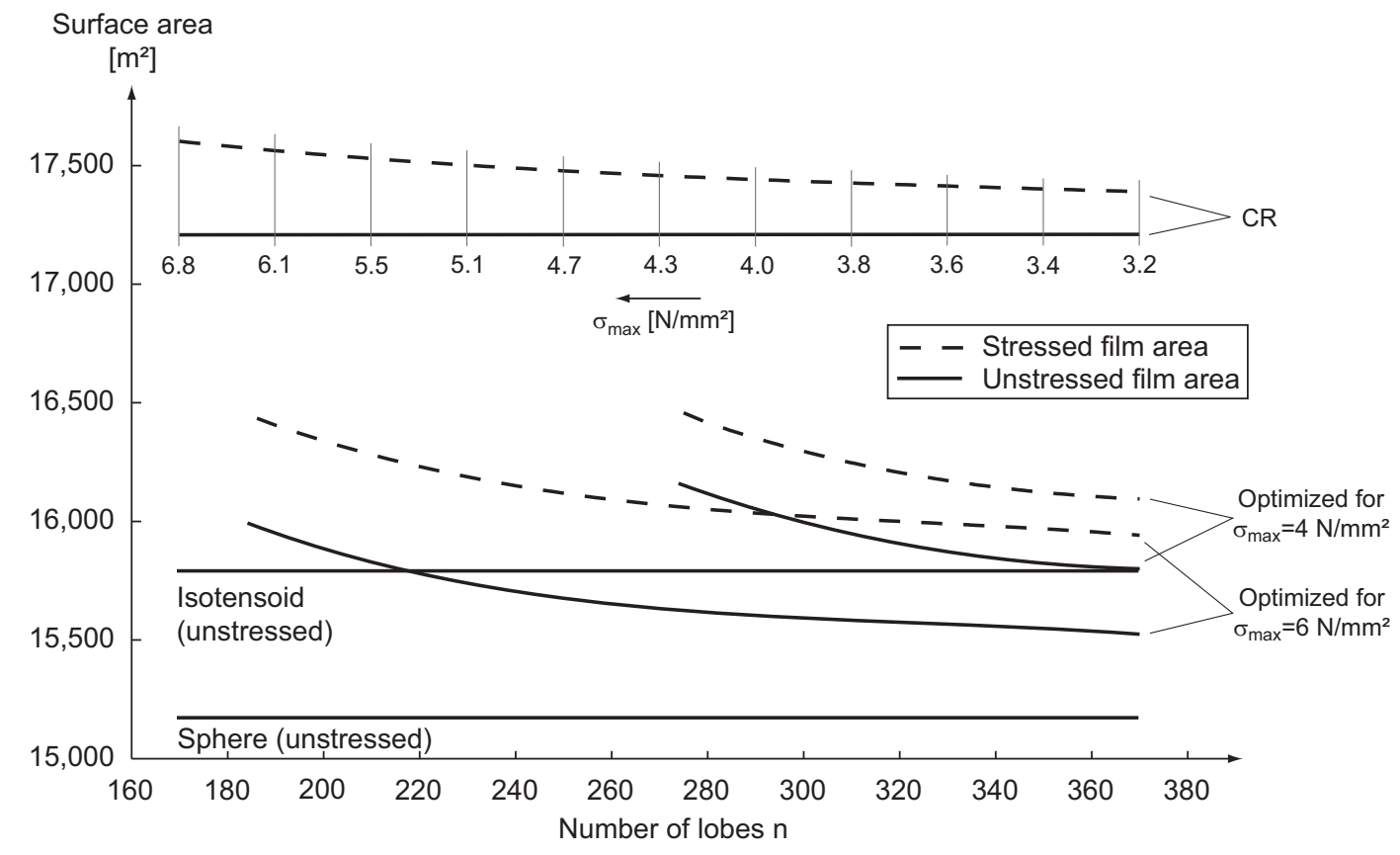

Figure 15. Surface area of CR and optimized designs before and after inflation with $p=200 \mathrm{~Pa}$.

\section{Thermal Loading Effects}

The manufacturing and operational temperatures of a balloon are different, hence thermal effects need to be taken into account. The thermal coefficient of the tendons is considerably smaller than that of the membrane and hence will be neglected, for simplicity.

A typical temperature to which a balloon is exposed during its mission is $\sim-30^{\circ} \mathrm{C}$. Assuming a mean temperature during manufacturing of $20^{\circ} \mathrm{C}$ the temperature difference results in $\Delta T=-50^{\circ} \mathrm{C}$. The effect on the membrane can be modeled by assuming an isotropic prestress of magnitude

$$
\sigma_{T}=\frac{\Delta T \alpha E}{1-\nu}
$$

where $\Delta T$ is the change of temperature and $\alpha$ is the linear coefficient of thermal expansion. This equation can be derived by computing the stresses that result in a fixed membrane, of any shape, from the thermal strains

$$
\varepsilon_{1}=\varepsilon_{2}=\Delta T \alpha .
$$

A new optimized cutting pattern that allows for both a pressure loading of $p=200 \mathrm{~Pa}$ and a thermal loading of $\Delta T=-50^{\circ} \mathrm{C}$ has been obtained by running the optimization algorithm. Figure 16 shows the 
stress distribution along the longitudinal centreline for both the CR lobe and the optimized lobe, due to $p=200 \mathrm{~Pa}$ and $\Delta T=-50^{\circ} \mathrm{C}$. It should be noted that, in both cases, the maximum von Mises stress is $8 \mathrm{~N} / \mathrm{mm}^{2}$. It can be seen that, for the optimized cutting pattern, both principal stresses are again more uniform along the arc-length. Again, it is found that given the number of lobes it is not possible to arbitrarily reduce the maximum von Mises stress in the membrane simply by increasing the width of the lobe cutting pattern. The reason for this is again the stress peak at the equator, which has been further increased by the thermal effects.

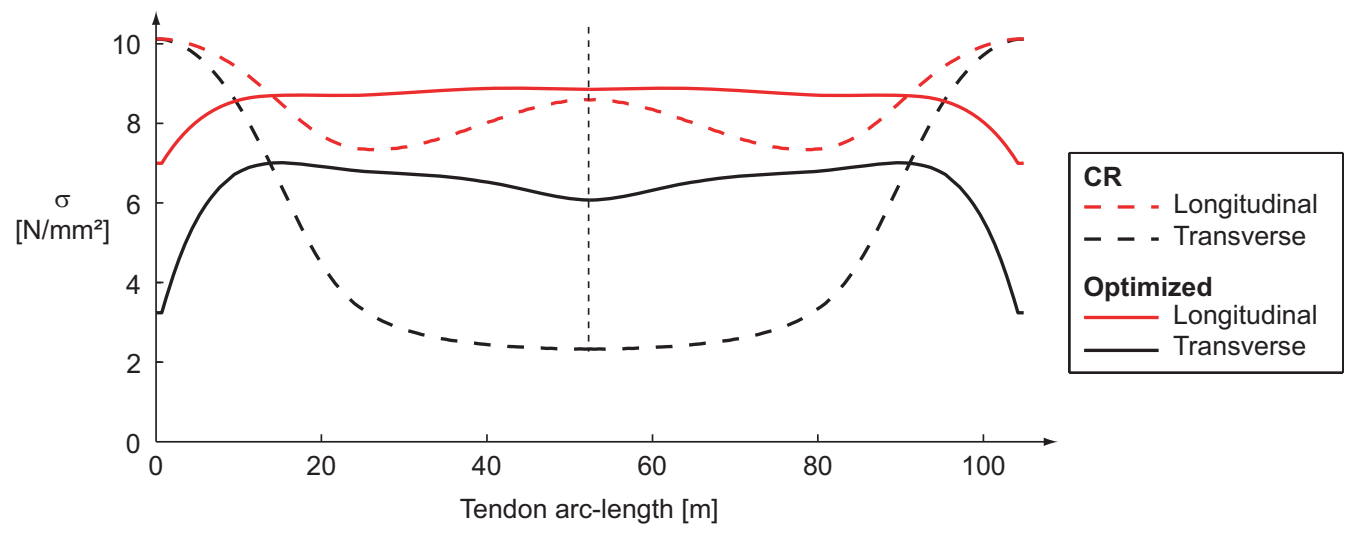

Figure 16. Principal stresses in longitudinal direction of CR and optimized flight-size balloon at $200 \mathrm{~Pa}$ and a membrane temperature of $\Delta T=-50^{\circ} \mathrm{C}$.

Figure 17 compares the shapes of the $\mathrm{CR}$ and optimized cutting patterns when loaded by $p=200 \mathrm{~Pa}$ and $\Delta T$ equal to either 0 or $=-50^{\circ} \mathrm{C}$. In Figure $17(\mathrm{a})$ it can be seen that both cutting patterns become flatter, i.e. the arc/cord length becomes smaller, when the thermal loading is removed. It can also be seen that the optimised pattern leads to a lobe up to $11 \%$ flatter although the peak stress is practically the same. Comparing Figure 17(a) with Figure 14(a) we see that the cutting pattern optimized for the thermal as well as pressure loading has larger arc/cord length near the apex of the balloon.

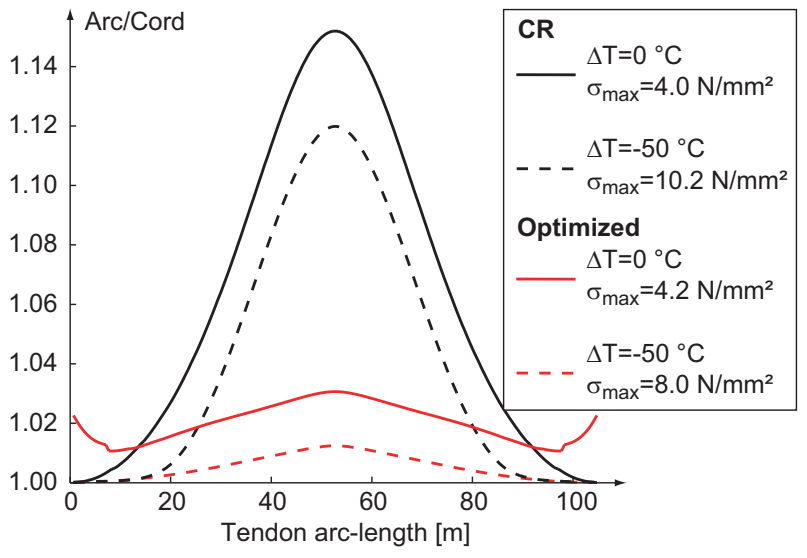

(a) Ratio between arc-length and cord-length.

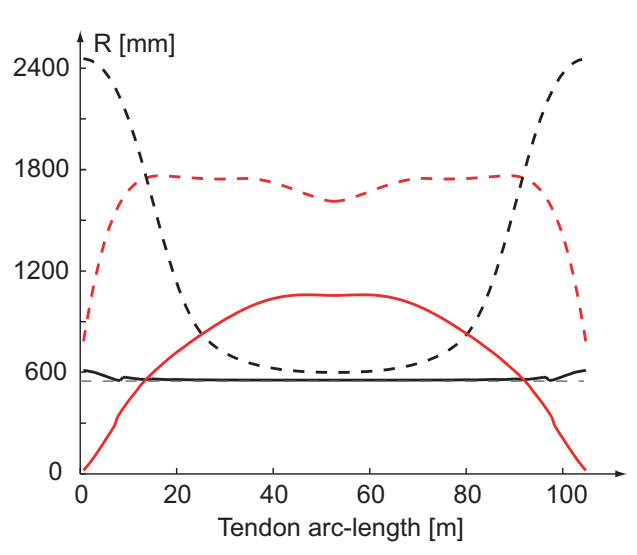

(b) Radius.

Figure 17. Comparison of CR and optimized cutting pattern at $p=200 \mathrm{~Pa}$.

Figure 18 shows the deformed and undeformed surface areas of balloons with CR and optimized cutting patterns, together with the maximum von Mises stresses that occur for $p=200 \mathrm{~Pa}$ and $\Delta T=50^{\circ} \mathrm{C}$. Again, it is found that the optimized cutting patterns have a considerably smaller surface area than the conventional CR designs, at comparable or even smaller maximum von Mises stresses. Due to the thermal effects, this time the inflated surface area is, depending on the number of lobes and stress constraint, smaller than the undeformed area. Therefore, in the plot the dotted lines lie below the solid lines, but again the change of surface area is related to the maximum allowed membrane stresses. Furthermore, the pressurized surface 
area, for a given maximum von Mises stress and the smallest possible number of lobes, is again constant.

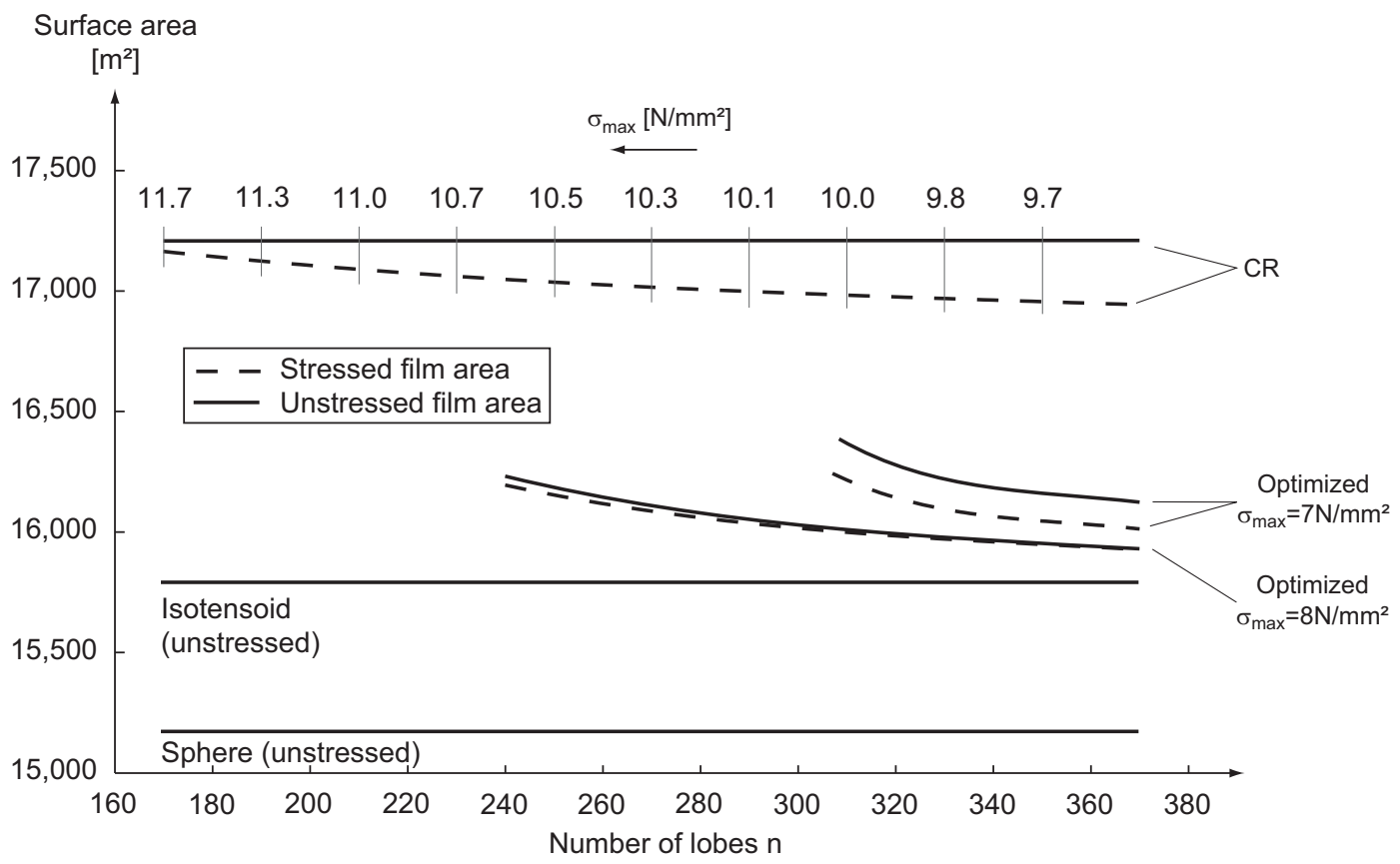

Figure 18. Surface area of CR and optimized designs before and after inflation with $p=200 \mathbf{P a}$ and $\Delta T=-50^{\circ} \mathbf{C}$.

Finally, Figure 19 compares the stress distribution in CR and optimized designs, with and without thermal loading, at a differential pressure of $p=200 \mathrm{~Pa}$. It should be noted that the optimized design results in a relatively uniform stress distribution, both with and without thermal loading. Furthermore, the maximum stress for the thermal loading is smaller than that observed for a standard CR design.

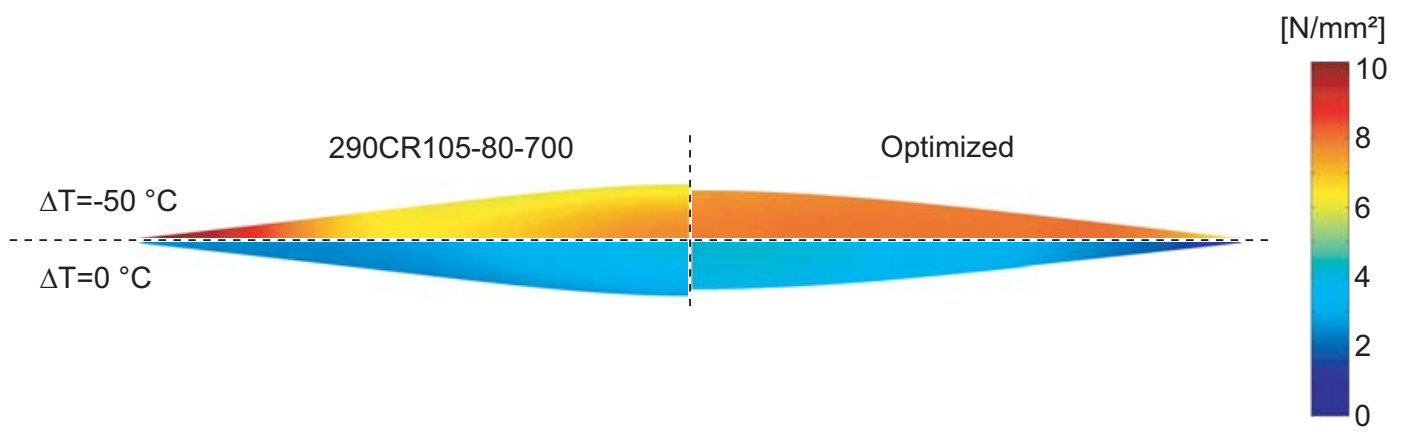

Figure 19. Stresses in $\mathbf{C R}$ and optimized cutting patterns for $p=200 \mathrm{~Pa}$ and $\Delta T=0,-50^{\circ} \mathbf{C}$.

\section{Conclusion}

It has been shown that the stability of pumpkin balloons can be considerably increased by optimizing the lobe cutting patterns. The maximum stability is reached if the surface area of the optimized lobe is minimized under a given stress constraint which results in a fully stressed design. One of the striking results is that the unstressed surface area is in some cases smaller than the area of an unlobed, rotationally smooth surface. This suggests a potential way of eliminating the shape instability problems that have affected ULDB balloons.

An important limitation of the present study, that still remains to be addressed, is the visco-elastic 
behaviour of the balloon film. This film has been modelled as time-independent and linear-elastic, and the value of the Young's Modulus that has been provided in Table 1 takes into account the visco-elastic deformation that is expected to take place over a period of 100 minutes, for an average stress level. The instantaneous modulus of the film is much higher and so the initial stresses in the balloon will also be much higher; they will then quickly decrease. These viscoelastic effects will need to be taken into account in future studies.

\section{Acknowledgments}

We thank Mr Rodger Farley (NASA Goddard Space Center), Dr Jim Rand (Winzen Engineering) and Dr David Wakefield (Tensys Dynamics Limited) for helpful discussions. This research was partially supported by the NASA Balloon Program Office, Program Monitor Mr Danny Ball (Columbia Scientific Balloon Facility). MP is a Research Fellow at St John's College, Cambridge.

\section{References}

\footnotetext{
${ }^{1}$ Calladine, C.R., Stability of the Endeavour balloon, In: Buckling of Structures, 1988, 133-149.

${ }^{2}$ Lennon, B.A., and Pellegrino S., Stability of Lobed Inflatable Structures, 41st AIAA/ASME/ASCE/AHS/ASC SDM Conference, AIAA 2000-1728, 2000.

${ }^{3}$ Wakefield D., Numerical Modelling of Pumpkin Balloon Instability, AIAA 5th ATIO and 16th Lighter-Than-Air System Technology and Balloon Systems Conferences, AIAA-2005-7445, 2005.

${ }^{4}$ Pagitz, M., and Pellegrino, S., Buckling Pressure of "Pumpkin" Balloons, International Journal of Solids and Structures, in press, 2007.

${ }^{5}$ Pagitz, M., and James, J., Symmetry Transformation Matrices for Structures, Proceedings of the Royal Society A: Mathematical, Physical and Engineering Sciences, in press, 2007.

${ }^{6}$ Bernstein, S.N., Démonstration du Théorème de Weierstrass fondée sûr le calcul dés Probabilités, Commun. Soc. Math. Khrakow, 1912.

${ }^{7}$ Zoutendijk, G., Methods of Feasible Directions, Elsevier, 1960.

${ }^{8}$ Vanderplaats, G.N., Numerical Optimization Techniques For Engineering Design, Vanderplaats Research \& Development, Inc., 2001.

${ }^{9}$ Karush, W., Minima of Functions of Several Variables with Inequalities as Side Constraints, Department of Mathematics, University of Chicago, 1939.

${ }^{10}$ Kuhn, H.W., and Tucker, A.W., Nonlinear programming, Proceedings of $2^{\text {nd }}$ Berkeley Symposium, 1951.

${ }^{11}$ Press, W.H., and Teukolsky, S.A., and Vetterling, W.T., and Flannery, B.P., Numerical Recipes in C ++ , Cambridge University Press, 2003.
} 